\title{
Neuroprotective effects of DHA in Alzheimer's disease models
}

\author{
Sabrina FLORENT-BÉCHARD \\ Violette KOZIEL \\ Jean-Luc OLIVIER \\ Thierry OSTER \\ Thierry PILLOT \\ Lipidomix (JE2482), ENSAIA \\ Institut National Polytechnique de Lorraine, \\ 15, rue du Bois de la Champelle, \\ 54505 Vandoeuvre-lès-Nancy, France \\ $<$ Thierry.Pillot@mtm.nancy.inserm.fr>
}

\begin{abstract}
Alzheimer's disease (AD) is a major public health concern in all developped countries. Although the precise cause of $A D$ is still unknown, a growing body of evidence supports the notion that soluble oligomers of amyloid $\beta$-peptide $(A \beta)$ may be the proximate effectors of synaptic injuries and neuronal death in the early stages of $A D$. AD patients display lower levels of docosahexaenoic acid (DHA, $\mathrm{C} 22: 6 ; n-3)$ in plasma and brain tissues as compared to control subjects of same age. Furthermore, epidemiological studies suggest that high DHA intake might have protective properties against neurodegenerative diseases. These observations are supported by in vivo studies showing that $\mathrm{DHA}$-rich diets limit the synaptic loss and cognitive defects induced by $A \beta$ peptide. Although the molecular basis underlying these neuroprotective effects remains unknown, several mechanisms have been proposed such as (i) regulation of the expression of potentially protective genes, (ii) activation of antiinflammatory pathways, (iii) modulation of functional properties of the synaptic membranes along with changes in their physicochemical and structural features. We recently demonstrated that DHA protects neurons from soluble A $\beta$ oligomer-induced apoptosis. Indeed, DHA pretreatment was observed to significantly increase neuronal survival upon $A \beta$ treatment by preventing cytoskeleton perturbations, caspase activation and apoptosis, as well as by promoting ERK-related survival pathways. These data suggest that DHA enrichment most likely induces changes in neuronal membrane properties with functional outcomes, thereby increasing protection from soluble $A \beta$ oligomers. Such neuroprotective effects could be of major interest in the prevention of $A D$ and other neurodegenerative diseases.
\end{abstract}

Key words: Alzheimer's disease, docosahexaenoic acid, neuronal membrane, neuroprotection, soluble A $\beta$ oligomers

the central nervous system on the development of $A D$. Docosahexaenoic acid (DHA, $\left.\mathrm{C} 22: 6^{\Delta 4,7,10,13,16,19} ; n-3\right)$ represents the longest and the most unsaturated fatty acid (FA) commonly found in biological systems and is mainly present in fish and algae. It is the major n-3 PUFA constituent of the neuronal membranes in the grey matter of the cerebral cortex and in retinal photoreceptor cells [6]. Because it is highly unsaturated, DHA is expected to increase the fluidity of neuronal membranes, thereby playing a role in various neurochemical processes in brain [7].

Given the enormous economical and societal burdens, there is an enormous medical need for the development of novel therapeutic strategies that target or even better prevent the molecular mechanisms leading to AD dementia. In this review, we wished to provide an overview on the beneficial effect of DHA on brain tissue in general and in the particular context of $A D$, leading to the idea that dietary DHA supplementation could be an efficient preventive strategy for delaying or preventing $A D$ and other neurodegenerative diseases.

\section{DHA, an essential fatty acid for the central nervous system}

DHA is the major $n-3$ PUFA constituent in the neuronal membranes, present in approximately $30-40 \%$ of the phospholipids of the gray matter of cerebral cortex and photoreceptor cells in the retina [6]. In the last trimester of fetal life and the first two years of childhood, the brain undergoes a period of rapid growth termed the "brain growth spurt". During this period, the need in this PUFA is dramatically elevated because of the increase in brain size and in relative DHA contents. Animal studies have demonstrated that reductions in perinatal brain DHA accrual are associated with deficits in neuronal arborisation, multiple indices of synaptic pathology including deficits in serotonin and mesocorticolimbic dopamine neurotransmission, neurocognitive deficits, elevated behavioral indices of anxiety, aggression and depression and decreased visual acuity [8]. In primates and humans, preterm delivery has been shown to be associated with the same troubles which can be reverted by $n-3$ PUFA supplementation [9]. After the perinatal brain development, DHA intake remains essential for the normal maintenance of brain functions including synaptic plasticity, neurotransmission and vision [10]. Because neurons lack the enzymes necessary for de novo DHA and arachidonic acid (AA, C20:4 $\left.4^{\Delta 5,8,11,14} ; n-6\right)$ synthesis, these FA are derived either directly from the diet or are mainly synthesized from the dietary precursors, $\alpha$-linolenic acid (ALA, C18:3 $\left.{ }^{\Delta 9,12,15} ; n-3\right)$ and linoleic acid (LA, $\left.\mathrm{C} 18: 2^{\Delta 9,12} ; n-6\right)$ in liver and in a minor way in cerebral endothelium or in astrocytes from where they are exported to neuronal cells [7] (figure 1). 


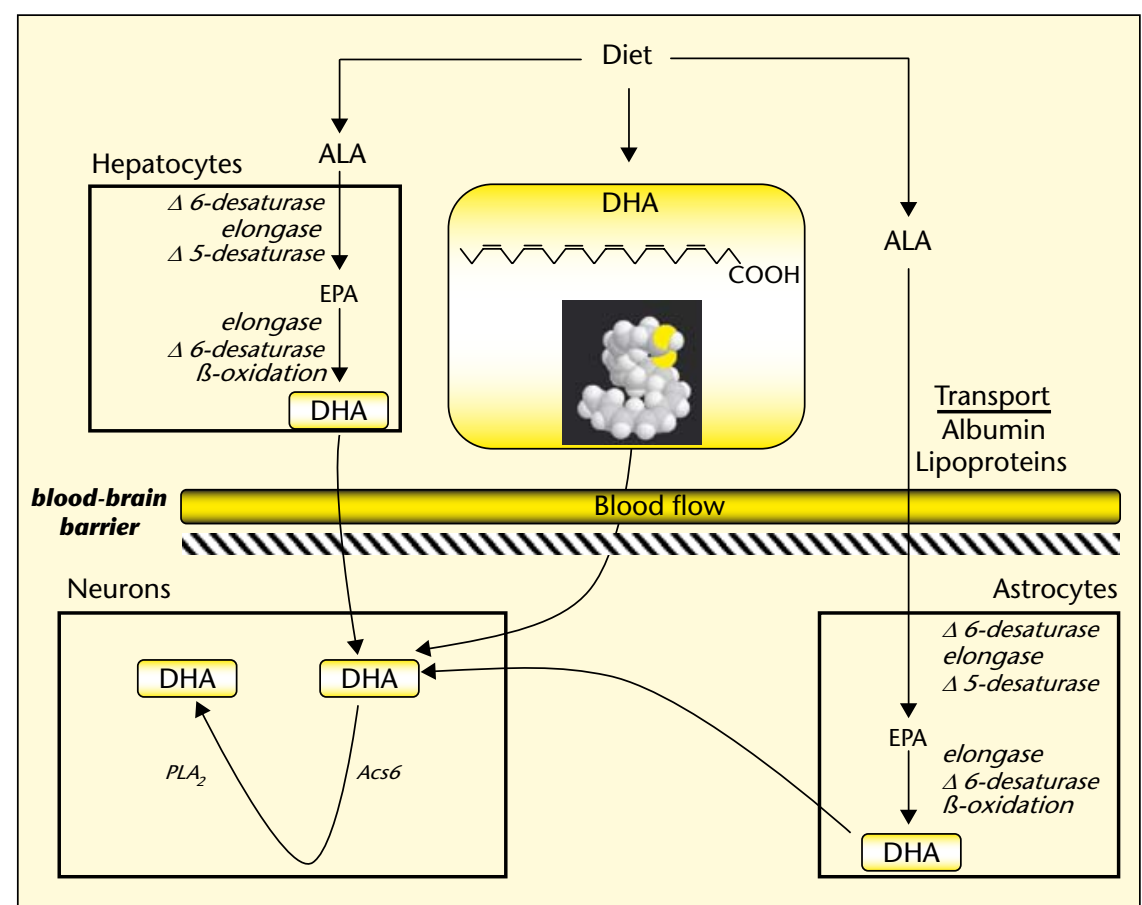

Figure 1. Dietary intake and synthesis pathway of docosahexaenoic acid. Fatty acids are derived either directly from the diet or are mainly synthesized from the dietary $n-3$ precursors (or incidentally in astrocytes) from where they are transported to the brain tissue. After incorporation by the cell, the turn-over of DHA involves a deacylation-reacylation cycle relying on selective phospholipase $A_{2}$ and acyltransferase such as Acs6.

\section{DHA and Alzheimer's disease}

Recent findings suggest a possible role of diet in age-related cognitive decline and impairments. Among the nutritional factors influencing $A D$ occurrence, moderate fish consumption as a proxi of $n$-3 PUFA intake was related to a reduced risk of impaired cognitive functions [11]. In the same way, a recent populationbased study among middle aged women suggests that dietary cholesterol and to a lesser extent saturated FA intake was associated with an increased risk of $A D$, while consumption of $n$-3 PUFA such as eicosapentaenoic acid (EPA ; C20:5 $\left.5^{\Delta 5,8,11,14,17}\right)$ and DHA was associated with a decreased risk of cognitive impairment, independently of differences in age, gender, education, smoking, total energy uptake and cardiovascular risk factor [12]. Importantly, lower contents of $n-3$ PUFA have also been measured in the plasma [13] as well as in the brain [14] of AD patients. Recent in vivo studies have reported that reduction of dietary $n-3$ PUFA in Tg2576 AD mouse model resulted in a loss of postsynaptic proteins and behavioral deficits, while a DHA-enriched diet could prevent these effects [15]. Furthermore, dietary DHA was not only shown to be protective against $A \beta$ production, accumulation and toxicity in Tg2576 mice [16] and AD model rats [17], but it could also ameliorate cognitive impairments in $A \beta$-infused rats [18]. This there-
For one simple molecule to affect so many seemingly unrelated processes, DHA must function at a fundamental level, common to most cells such as transcription events, membrane structure and functions and/or signal transduction (figure 2) [19]. A nutrigenomic approach with high-density microarrays revealed changes in the expression of brain genes in response to different PUFA-enriched diets. It emphasised significant changes in the expression of several genes, as demonstrated by altered transcription of various genes, including that encoding the $A \beta$-scavenger transthyretin, in hippocampus of aged rats fed with fish oil [20]. Some reports have concluded that DHA or fish oil supplementation resulted in antioxidant effects in hippocampus and cortex of an $A D$ model rat [17] as well as in rat hippocampal cultures exposed to glutamate [21]. It could then be suggested that the preventive effect described in epidemiological studies could be due to antioxidant properties of this FA.

We recently demonstrated that DHA strongly protects rat cortical neurons from soluble $A \beta$ oligomer-induced neurodegeneration and apoptosis (figure 3) [22]. It is noteworthy that DHA prevents soluble $A \beta$ oligomer-mediated cytosketelon perturbation [23], as well as activation of both neutral and acidic sphingomyelinases [24]. We also reported that DHA pretreatment preserves the capacity of neurons to phosphorylate ERK1/2 upon exposure to soluble $A \beta$ (figure 4). The enrichment of membrane in DHA has thus proven its crucial interest in maintaining a sufficient rate of these phosphorylated/active proteins, whose associated survival pathways are thereby promoted in neurons. This suggests that not only DHA is required as an essential membrane constituent, but it also likely acts as a sensitive switch of major importance for modulating most signaling pathways including cell apoptosis and survival. Further experiments are required to iden-

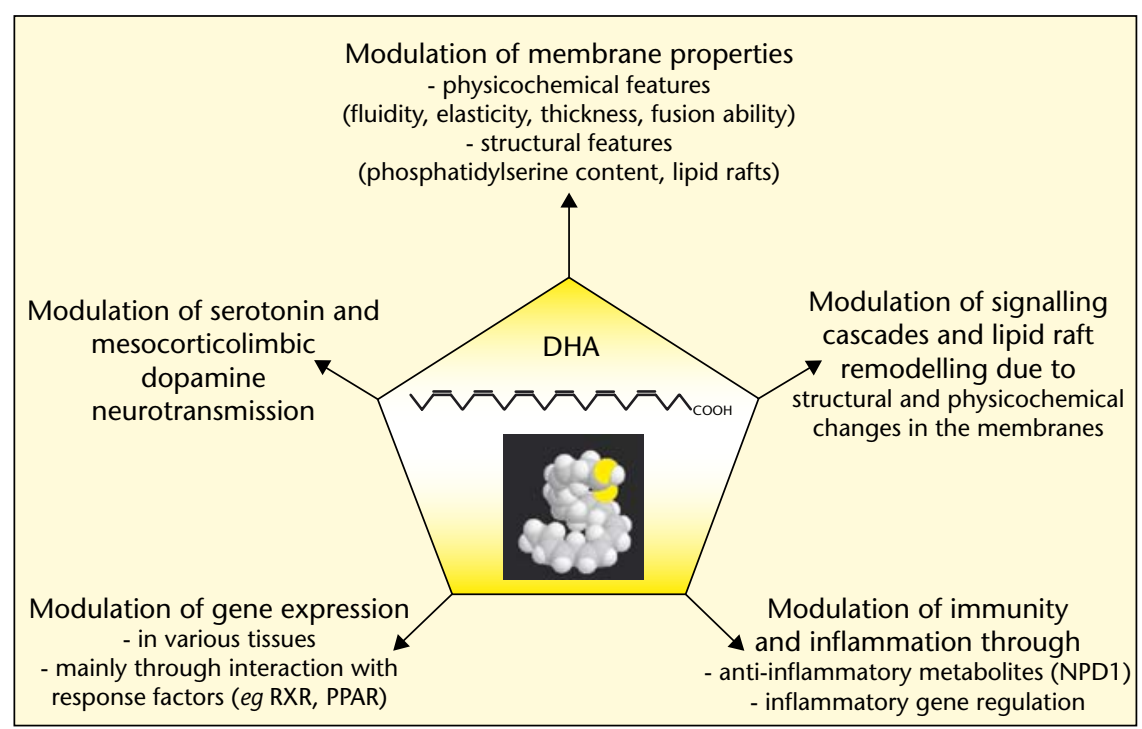

Figure 2. Implication of DHA in neuronal integrity and survival. 


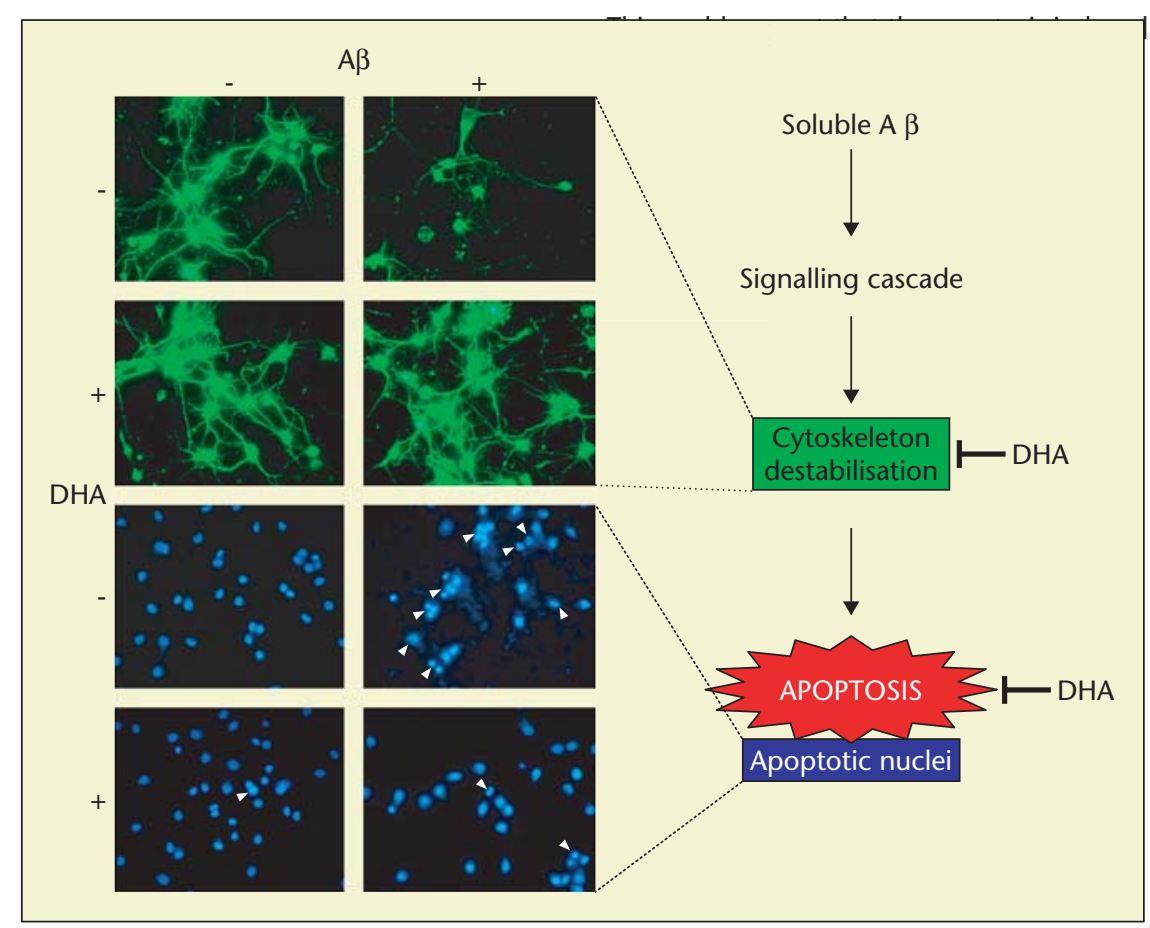

Figure 3. Docosahexaenoic acid prevents neuronal apoptosis induced by soluble $A \beta$ oligomers. DHA prevents both cytoskeleton perturbations and apoptosis induced by $A \beta$ soluble oligomers as demonstrated by a cytoskeleton integrity analysis after $\beta$-tubulin labelling and by a visualization after 4,6-diamidino-2-phenylindole staining respectively [22]. by $A \beta$ oligomers involves structural and qualitative changes in lipid rafts, which are prevented after DHA pretreatment. These changes are expected to have functional outcomes in terms of regulating neuronal signaling cascades.

\section{Conclusion}

Diet strongly influences the incidence and outcome in major age-related disease including cardiovascular diseases, cancer and dementia such as AD. Several epidemiological and experimental data suggest that DHA intake and enrichment in neuronal membranes could provide a substantial protective effect against these devastating pathologies, which undoubtedly represents one of the most promising preventive approaches to develop with the aim to prevent or to delay the onset of $A D$. Different ways of action could contribute to the neuroprotective as well as neurotrophic properties of DHA. Further studies are still necessary to identify the preferential mechanism(s) with the view to optimize this approach and to improve its interest on rational and scientifically established bases instead of nutritional or nutraceutical treatments that are still often proposed on empirical considerations. Also, it is likely that the neuroprotective effects of DHA might be further enhanced by coupling the FA tify the proteins and domains in the plasma membrane that could act as protective sensors able to induce the antiapoptotic response triggered by DHA enrichment in neurons. Focusing on architectural changes PUFA enrichment could induce in neuronal plasma membrane, the most interesting hypothesis to explain neuroprotective effects of DHA might concern its impact on lipid rafts, defined as compositionally distinct platforms for compartmentalizing dynamically regulated signaling assemblies at the plasma membrane. DHA and PUFA enrichment is known to be accompanied by lateral phase separation and local lipid redistribution, leading to membrane remodeling [25]. In our experimental model, protection of rat cortical neurons from $A \beta$-induced apoptosis was obtained by supplementing the medium with nanomolar DHA concentrations, likely resulting in DHA enrichment of specific phospholipids species or membrane microdomains. We thus hypothesized that subtle changes could have occurred in rafts, affecting their lipid as well as protein components. Accordingly, we studied the raft-specific ganglioside M1 and flotillin by immunocytochemistry and showed that exposition of cortical neurons to soluble $A \beta$ peptide leads to a membrane disorganisation, whereas neurons pretreated with DHA still exhibit intense fluorescence labeling (figure 4).

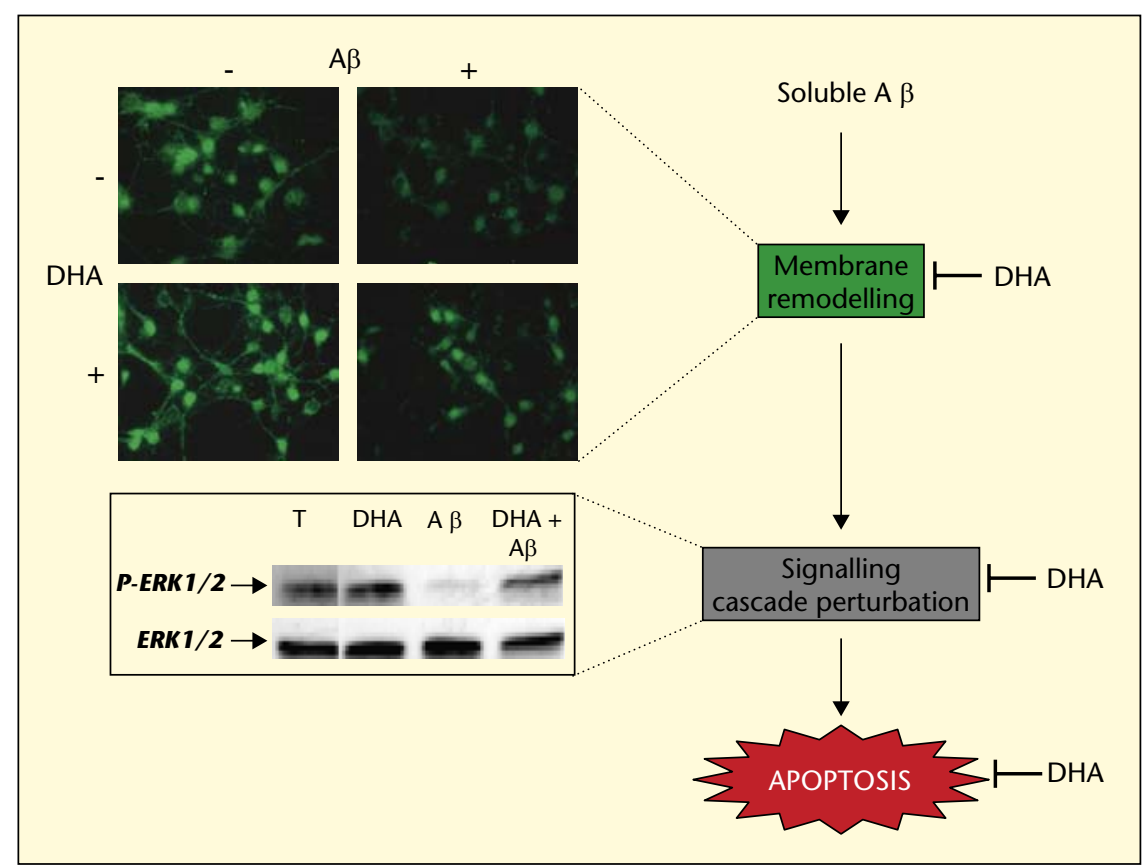

Figure 4. DHA might prevent apoptosis through fine membrane remodeling. Interaction of $A \beta$ with plasma membrane of cortical neurons might represent an early event in a cascade leading to neurodegeneration. This interaction is not prevented by DHA treatment as shown by Florent et al. [22] but preliminary results suggest that DHA might not only prevent the initiation of apoptotic cascade but also maintain survival signalling through fine membrane remodelling. The maintenance of the ERK1/2 signalling pathway is here presented as an example of these neuroprotective effects. 
to anti-inflammatory and/or antioxidant molecules such as polyphenols, given that many papers have also associated the consumption of vegetables and fruits with a lower $A D$ risk [26]. Original DHA-based formulations might therefore provide essential health benefits in preventing $A D$ for which no disease-modifying therapies are currently available.

\section{REFERENCES}

1. SELKOE DJ. Alzheimer's disease is a synaptic failure. Science 2002; 298: 789-91.

2. DROUET B, PINCON-RAYMOND M, CHAMBAZ J, PILLOT T. Molecular basis of Alzheimer's disease. Cell Mol Life Sci 2000; 57: 705-15.

3. SPONNE I, FIFRE A, DROUET B, et al. Apoptotic neuronal cell death induced by the non-fibrillar amyloid- $\beta$ peptide proceeds through an early reactive oxygen species-dependent cytoskeleton perturbation. / Biol Chem 2003; 278: 3437-45.

4. KRIEM B, SPONNE I, FIFRE A, et al. Cytosolic phospholipase $\mathrm{A}_{2}$ mediates neuronal apoptosis induced by soluble oligomers of the amyloid- $\beta$ peptide. FASEB / 2005; 19: 85-7.

5. PILLOT T, DROUET B, QUEILLE $S$, et al. The nonfibrillar amyloid $\beta$-peptide induces apoptotic neuronal cell death: involvement of its C-terminal fusogenic domain. I Neurochem 1999; 73: 1626-34.

6. LAURITZEN L, HANSEN HS, JORGENSEN MH, MICKAELSEN KF. The essentiality of long chain $n 3$ fatty acids in relation to development and function of the brain and retina. Prog Lipid Res 2001; 40: 1-94

7. HORROCKS LA, FAROOQUIAA. Docosahexaenoic acid in the diet: its importance in maintenance and restoration of neural membrane function. Prosatglandins Leukot Essent Fatty Acids 2004; 70: 361-72.

8. MCNAMARA RK, CARLSON SE. Role of omega-3 fatty acids in brain development and function: potential implications for the pathogenesis and prevention of psychopathology. Prostaglandins Leurkot Essent Fatty Acids 2006; 75: 329-49.
9. CARRIE I, CLEMENT M, DE JAVEL D, FRANCES H, BOURRE JM. Phospholipid supplementation reverses behavioural and biochemical alterations induced by $n-3$ polyunsaturated fatty acid deficiency in mice. / Lipid Res 2000; 41: 473-80.

10. MCCANN IC, AMES BN. Is docosahexaenoic acid, an $n-3$ long-chain polyunsaturated fatty acid, required for development of normal brain function? An overview of evidence from cognitive and behavioral tests in humans and animals. Am / Clin Nutr 2005; 82: 281-95.

11. MORRIS MC, EVANS DA, BIENIAS JL, et al. Consumption of fish and $n-3$ fatty acids and risk of incident Alzheimer disease. Arch Neurol 2003; 60: 940-6.

12. KALMIJN S, VAN BOXTEL MPJ, OCKE M, VERSCHUREN WMM, KROMHOUT D, LAUNER LI. Dietary intake of fatty acids and fish in relation to cognitive performance at middle age. Neurology 2004; 62: 275-80.

13. TULLY AM, ROCHE HM, DOYLE R, et al. Low serum cholesteryl ester-docosahexaenoic acid levels in Alzheimer's disease: a case-control study. Br J Nutr 2003; 89: 483-9.

14. SODERBERG M, EDLUND C, KRISTENSSON K DALLNER G. Fatty acid composition of brain phospholipids in aging and in Alzheimer's disease. Lipids 1991; 26: 42-5.

15. CALONF, LIM GP, YANG F, et al. Docosahexaenoic acid protects from dendritic pathology in an Alzheimer's disease mouse model. Neuron 2004; 43: 633-45.

16. LIM GP, CALON F, MORIHARA T, et al. A diet enriched with the $\omega$-3 fatty acid docosahexaenoic acid reduces amyloid burden in an aged Alzheimer mouse model. J Neurosci 2005; 25: 3032-40.

17. HASHIMOTO M, HOSSAIN S, SHIMADAT, OKA II, SHIDO O. Docosahexaenoic acid provides protection from impairment of learning ability in Alzheimer's disease model rats. I Neurochem 2002; 81: 1084-91.
18. HASHIMOTO M, TANABE Y, FUJII Y, KIKUTA T, SHIBATA H, SHIDO O. Chronic administration of docosahexaenoic acid ameliorates the impairment of spatial cognition ability in amyloid $\beta$-infused rats. / Nutr 2005; 135: 549-55.

19. FLORENT-BECHARD $S$, OLIVIER IL, PILLOTT, OSTER T. Towards a nutritional approach for prevention of Alzheimer's disease. Biochemical and cellular aspects. I Neurol Sci 2007; doi: 10.1016/j.jns.2007.06.046.

20. KITAJKA K, PUSKAS LG, ZVARA Jr. L, BARCELOCOBLIJN G, YEO YK, FARKAS T. The role of $n-3$ polyunsaturated fatty acids in brain: modulation of rat brain gene expression by dietary $n-3$ fatty acids. Proc Natl Acad Sci USA 2002; 99: 2619-24.

21. WANG X, ZHAOX, MAOZ, WANG XM, LIU ZL. Neuroprotective effect of docosahexaenoic acid on glutamate-induced cytotoxicity in rat hippocampal cultures. Neuroreport 2003; 14: 2457-61.

22. FLORENT S, MALAPLATE-ARMAND C, YOUSSEF I et al. Docosahexaenoic acid prevents neuronal apoptosis induced by soluble amyloid- $\beta$ oligomers. / Neurochem 2006; 96: 385-95.

23. FIFRE A, SPONNEI, KOZIEL $V$, et al. Microtubule-associated protein MAP1A, MAP1B, and MAP2 proteolysis during soluble amyloid $\beta$-peptide-induced neuronal apoptosis. Synergistic involvement of calpain and caspase-3. J Biol Chem 2006; 281: 229-40.

24. MALAPLATE-ARMAND C, FLORENT-BECHARD S, YOUSSEF I, OLIVIER IL, OSTERT, PILLOTT. Soluble oligomers of amyloid- $\beta$ peptide induce neuronal apoptosis by activating a $C P L A_{2}-$ dependent sphingomyelinase-ceramide pathway. Neurobiol Dis 2006; 23: 178-89.

25. WASSALL SR, BRZUSTOWICZ MR, SHAIKH SR, CHEREZOV V, CAFFREY M, STILLWELL W. Order from disorder, corralling cholesterol with chaotic lipids. The role of polyunsaturated lipids in membrane raft formation. Chem Phys Lipids 2004; 132: 79-88.

26. RAMASSAMY C. Emerging role of polyphenolic compounds in the treatment of neurodegenerative diseases: a review of their intracellular targets. Eur J Pharmacol 2006; 545: 51-64. 